\title{
Radiación fotosintéticamente activa y propiedades fisico-químicas en suelos forestales con y sin incendio
}

\author{
Carlos César Maycotte Morales ${ }^{1}$ \\ Alejandro Velázquez Martínez ${ }^{2}$ \\ J. Jesús Vargas Hernández ${ }^{2}$ \\ Antonio Trinidad Santos ${ }^{3}$ \\ Miguel Ángel Musálem Santiago ${ }^{4}$ \\ Gil Vera Castillo ${ }^{5}$
}

\begin{abstract}
RESUMEN
Se evaluó el efecto del fuego sobre el incremento de la radiación fotosintéticamente activa y las propiedades físico-químicas en suelos quemados y no quemados. La evaluación incluyó el período de finales del invierno de 1999 a inicios de la primavera del 2000, en el ejido "El Reparo" en Zacualtipán, Hidalgo, en un bosque de Pinus patula que fue afectado por un incendio en la primavera de 1998. Los datos indicaron que existe un incremento sustancial de radiación en los sitios quemados en comparación con aquellos sitios no quemados y que la incidencia de luz es homogénea a lo largo de la pendiente. El fuego no modificó la textura, $\mathrm{pH}$, concentración del nitrógeno inorgánico, potasio, magnesio, hierro, cobre, zinc, manganeso y de la capacidad de intercambio catiónico. Al final del período de evaluación, la materia orgánica, potasio y magnesio aumentaron en los sitios quemados, alcanzando valores similares a los existentes en los sitios no quemados. Al final del estudio, el fuego modificó el patrón espacial de distribución de algunos nutrimentos como el hierro, zinc, manganeso y cobre, los cuales al inicio no presentan un patrón definido y, al final, tienden a incrementarse hacia los sitios de menor elevación.
\end{abstract}

PALABRAS CLAVE:

Incendio, nutrimentos, Pinus patula, radiación fotosintéticamente activa.

\begin{abstract}
The effect of fire on the increment of photosynthetic active radiation and physical and chemical properties in soils was evaluated during late winter 1999 and early spring 2000 in Zacualtipán, Hidalgo, in a Pinus patula forest that was affected by fire in the spring of 1998. The data showed that radiation increased drastically in the burned stand, considering that radiation incidence was homogeneous along the slope in both stands. Fire did not modify soil texture, $\mathrm{pH}$, inorganic nitrogen, potassium, magnesium, iron, copper, zinc, manganese and cation exchange capacity. After a six month period, soil organic matter, potassium and magnesium increased again up to similar levels as in the unburned stand. Fire also modified the spatial pattern of some ions such as iron, zinc, manganese, and copper. Initially, these ions did not show a spatial trend in concentration, but after a six month period, they tended to increase their concentration in the lower elevation sites.
\end{abstract}

KEY WORDS:

Fire, nutrients, Pinus patula, photosynthetic active radiation. 


\section{INTRODUCCIÓN}

El incendio siempre ha sido un factor natural muy importante para la configuración del ambiente. Presenta una influencia tanto en las características de las especies como en las características y procesos del ecosistema, tal como el ciclo del carbono, el agua, los nutrimentos, la productividad y diversidad (Spurr y Barnes, 1980; Rodríguez, 1996).

Uno de los mayores cambios que se presenta en el bosque posterior a un disturbio, es el incremento sustancial de luz. La modificación en la intensidad lumínica tiene repercusiones importantes sobre el funcionamiento de la comunidad, debido a que provoca cambios drásticos en la tasa de fotosíntesis, productividad primaria neta, temperatura del suelo y del aire, disponibilidad de agua y actividad de los microorganismos del suelo (Dai, 1996). Esto se debe a que los disturbios provocan aperturas del dosel y promueven que el piso forestal reciba una mayor cantidad de luz solar en forma directa. Por lo tanto, la capacidad de germinación, supervivencia y crecimiento de las plantas tolerantes y no tolerantes a la sombra generalmente se correlaciona con el tamaño del claro (Canham et al., 1990; Stewart et al., 1991; Sipe y Bazzaz, 1995; Smith et al., 1997; Gray y Spies, 1997).

En condiciones no alteradas, la luz que alcanza al piso forestal puede variar ampliamente en cantidad y calidad. En cantidad, se puede recibir desde menos de $1 \%$ bajo rodales muy densos, hasta un $100 \%$ en grandes aperturas del bosque. Estas aperturas permiten que los árboles forestales se establezcan con mayor rapidez, ya que la apertura del dosel tenderá a cerrarse a través del tiempo. Aunque los árboles forestales alcanzan el punto de compensación de luz en cantidades del $1 \%$ al $2 \%$ de luz solar directa, se requiere al menos un $20 \%$ de intensidad luminosa sobre períodos largos para asegurar una buena supervivencia de las plántulas. El crecimiento de la raíz se ve seriamente afectado si éstas crecen en niveles bajos de luz directa, aún tratándose de especies muy tolerantes (Poulson y Platt, 1989; Canham et al., 1990; Smith et al., 1997).

Los incendios forestales también ocasionan una gran pérdida de nutrimentos, ya sea a través de la reducción de biomasa o de los residuos orgánicos que se encuentran en el suelo. Estos nutrimentos se pierden por volatilización, lavado superficial y por lixiviación. El nitrógeno (N) y el azufre (S) se volatilizan a temperaturas relativamente bajas. Los ecosistemas con grandes cantidades de nutrimentos en el suelo superficial pueden experimentar pérdidas catastróficas de nutrimentos. Por ejemplo, del $50 \%$ al $70 \%$ de $\mathrm{N}$ y otros elementos (ocasionalmente hasta el $80 \%$ ) pueden perderse durante incendios de gran intensidad (Waring y Schlesinger, 1985; Landsberg y Gower, 1997).

Sin embargo, el fuego frecuentemente incrementa la disponibilidad de algunos nutrimentos, a pesar de las grandes pérdidas vía lixiviación y volatilización, lo que no necesariamente mejora la calidad del sitio (Kozlowski et al., 1991). Si los minerales en las cenizas son lixiviados dentro del suelo y son absorbidos por el sistema radical, la calidad del sitio se mejora temporalmente. Pero si estos minerales son lixiviados bajo la zona radical o son lavados de la superficie del suelo y removidos a través del flujo de agua, la calidad del sitio se reduce (Kozlowski et al., 1991). Pritchett (1986) y Attiwill y Adams (1993) señalan que la topografía puede tener una influencia directa sobre la distribución de nutrimentos en la localidad, la cual se incrementa después de que los bosques han sufrido un disturbio.

\section{OBJETIVO}

Con base en lo anterior, el objetivo de este trabajo es evaluar el efecto del incendio sobre la radiación fotosintéticamente activa y propiedades fisico- 
químicas del suelo en rodales forestales quemados y no quemados, así como su dinámica estacional.

\section{MATERIALES Y MÉTODOS}

El estudio se realizó en el ejido "El Reparo" del municipio de Zacualtipán de Ángeles, en el Estado de Hidalgo. La zona se ubica en la Sierra Madre Oriental, subprovincia Sierra Zacualtipán, conocida localmente como Carso Huasteco. Las coordenadas geográficas corresponden a $20^{\circ} 37^{\prime} 34,05^{\prime \prime}$ de latitud Norte y $98^{\circ} 35^{\prime}$ $54,86^{\prime \prime}$ de longitud Oeste a una altura sobre el nivel del mar de $2100 \mathrm{~m}$, aproximadamente. El clima es templado húmedo. La precipitación media anual es de $1853 \mathrm{~mm}$ y la temperatura media anual es de $12,4^{\circ} \mathrm{C}$ (García, 1988; INEGI, 1996).

\section{Sitios de muestreo}

La zona de estudio consta de dos áreas de corta de Pinus patula, de 0,21 ha cada una; en una de éstas se presentó un incendio en 1998. Ambas fueron sometidas a la corta de repoblación de árboles padre en 1995 y presentan una pendiente de $45 \%$ en exposición sur. En estas áreas se establecieron dos transectos, uno en el área quemada y otro en la que no presentó este tipo de disturbio, ambos con dirección Norte-Sur.

En cada uno de estos transectos se establecieron 7 sitios de muestreo con una altura sobre el nivel del mar de 2090 m en el sitio que se encuentra en la parte más alta de la pendiente y de $2030 \mathrm{~m}$ en el sitio más bajo de la misma. Estos sitios fueron de forma circular con una superficie de $40 \mathrm{~m}^{2}$ cada uno y $50 \mathrm{~m}$ equidistantes uno de otro (Stein, 1992; Olvera et al., 1996; Eibl et al., 1998), existiendo una diferencia en altura de $10 \mathrm{~m}$ entre los sitios.

\section{Variables evaluadas}

La radiación fotosintéticamente activa se determinó por medio de un radiómetro/fotómetro/quantum modelo $\mathrm{LI}$ COR 189 en cada uno de los sitios de muestreo. El radiómetro se colocó a nivel del suelo de forma paralela a la pendiente, con el propósito de cuantificar la radiación que llegaba directamente al suelo. Las mediciones se efectuaron de manera regular entre las 12 y 14 horas una vez al mes, durante el período del 3 de abril de 1999 al 30 de marzo de 2000.

En cada ocasión, se trató de hacer las mediciones en un día soleado, sin embargo, debido a las condiciones ambientales que existen en la región, especialmente en lo que respecta a la presencia de niebla y nubosidad, esto fue imposible en ciertas épocas del año. Considerando esta situación, se aseguró que las condiciones de homogeneidad fueran estables en los dos transectos al momento de la medición.

Dado que el radiómetro evalúa la radiación recibida en un área de $3 \mathrm{~cm}^{2}$; en cada ocasión se tomó la lectura de 3 puntos distintos de cada sitio, separados $3 \mathrm{~m}$ entre sí, en forma sistemática; estos valores se promediaron para obtener la intensidad luminosa en cada sitio. Las unidades en que se determinó esta variable fueron $\mu \mathrm{mol} \mathrm{s}{ }^{-1} \mathrm{~m}^{-2}$ de manera instantánea.

Para evaluar la concentración de nutrimentos en el suelo se utilizó un muestreo sistemático. De cada sitio se extrajo una muestra de suelo (Peck y Melsted, 1973; Alcántara et al., 1992; Etchevers et al., 1992) a través de un muestreador de suelos Forestry Suppliers Bucket Auger de 3 plg de diámetro. Las muestras se tomaron tres veces al año, al inicio de la estación de crecimiento, al inicio de la época de lluvias y al finalizar la época de crecimiento. 
El muestreo se realizó en los primeros $5 \mathrm{~cm}$ de profundidad, tomando la muestra inicial en el centro del sitio y las dos posteriores en puntos situados a $3 \mathrm{~m}$ equidistantes a los lados del centro; es decir, que tanto la radiación como las características de los suelos se evaluaron en dirección Este-Oeste, en el sentido perpendicular a la pendiente.

La profundidad de muestreo es la recomendada por varios autores para analizar los cambios que suceden en el suelo ocasionados por las altas temperaturas que se alcanzan en los incendios naturales (Pritchett 1986; Swift et al., 1993; Knoepp y Swank, 1993; Elliot y Vose, 1993; Rodríguez, 1996; USDA, 1996). Se sabe que estas condiciones no son similares a las establecidas en la zona de estudio y a pesar de no contar con los registros de temperatura alcanzada por el incendio, se decidió considerar esta profundidad para los fines del estudio.

Las muestras de suelo se analizaron en el Laboratorio Central Universitario del Departamento de Suelos de la Universidad Autónoma Chapingo y en Laboratorio del Instituto de Ciencias de la Tierra de la Universidad Autónoma del Estado de Hidalgo, para las siguientes determinaciones, de acuerdo con las indicadas por Tan (1996): el pH se determinó por medio de un potenciómetro relación sueloagua $1: 2$ en $\mathrm{Ca} \mathrm{Cl}_{2} \quad 0,01 \mathrm{M}$. La materia orgánica (\%) por la técnica Walkley y Black en $\mathrm{K}_{2} \mathrm{Cr}_{2} \mathrm{O}_{7} \quad 1 \mathrm{~N}$. El nitrógeno inorgánico ( $\mathrm{mg} \mathrm{kg}^{-1}$ ) fue extraído con cloruro de potasio $2 \mathrm{~N}$ y determinado por arrastre de vapor Kjeltec Auto Analyzer 1030. El fósforo $\left(\mathrm{mg} \mathrm{kg}^{-1}\right)$ por medio del método Olsen. El potasio $\left(\mathrm{mg} \mathrm{kg}^{-1}\right)$ fue extraído en acetato de amonio $1,0 \mathrm{~N} \mathrm{pH}$ 7,0 relación 1:20 y determinado por espectrofotometría de emisión de flama. El calcio $\left(\mathrm{mg} \mathrm{kg}^{-1}\right)$ y el magnesio $\left(\mathrm{mg} \mathrm{kg}^{-1}\right)$ fueron extraídos en acetato de amonio $1,0 \mathrm{~N} \mathrm{pH} \mathrm{7,0} \mathrm{relación} \mathrm{1:20} \mathrm{y} \mathrm{determinados}$ por espectrofotometría de absorción atómica. El hierro, cobre, zinc y manganeso ( $\mathrm{mg} \mathrm{kg}^{-1}$ ) fueron extraídos con DTPA relación 1:4 y determinados por espectrofotometría de absorción atómica. La capacidad de intercambio cationico $\left(\mathrm{cmol}_{(+)} \mathrm{kg}^{-1}\right)$ fue determinada por acetato de amonio $1 \mathrm{~N}$ pH 7,0 centrifugación Kjeltec Auto Analyzer 1030 y la textura por el método del hidrómetro de Bouyoucos.

\section{Análisis estadístico}

Las diferencias en la cantidad de radiación fotosintéticamente activa y en las propiedades físico-químicas entre los transectos y los cambios estacionales, se realizaron mediante el análisis de varianza, comparando las fechas de evaluación.

Respecto al efecto de la posición de los sitios a lo largo de la pendiente, se realizó un análisis de varianza para determinar las diferencias existentes a lo largo de la pendiente y, posteriormente, se realizó un análisis de regresión para determinar la tendencia de las variables a lo largo de cada transecto a través del período de evaluación (SAS,1997). Estos análisis se realizaron por separado y en forma conjunta para determinar las diferencias posibles entre las fuentes de variación que fueron los transectos, las fechas y la interacción de estas dos variables.

En los análisis de regresión, la posición en la pendiente fue la variable independiente dentro de la ecuación. Las comparaciones de medias entre tratamientos (transectos y fechas) se hicieron considerando un nivel de significancia de $p<0,05$. En las ecuaciones de regresión se consideró que el coeficiente de regresión fuera significativamente diferente de cero (con $p<0,05)$ y que el coeficiente de determinación $\left(R^{2}\right)$ fuera superior a 0,5 .

\section{RESULTADOS}

\section{Radiación fotosintéticamente activa}

La cantidad de radiación que llegó al piso forestal en el transecto quemado es sustancialmente mayor a la que se 
presentó en el transecto no quemado. Esta diferencia en cantidad fue estadísticamente significativa ( $p=0,0001)$. Las diferencias en intensidad luminosa variaron a lo largo del período de evaluación (Fig. 1), posiblemente por las fluctuaciones asociadas a las condiciones ambientales en la zona. Así, en los sitios quemados se presentan valores que fluctúan desde los $590 \mu \mathrm{mol} \mathrm{s}{ }^{-1} \mathrm{~m}^{-2}$ en el mes de octubre, hasta $1950 \mu \mathrm{mol} \mathrm{s}{ }^{-1} \mathrm{~m}^{-2}$ en el mes de abril. En los sitios no quemados, se presenta la misma tendencia, aunque los valores varían desde $100 \mu \mathrm{mol} \mathrm{s} \mathrm{s}^{-1} \mathrm{~m}^{-2}$ en el mes de febrero hasta $597 \mu \mathrm{mol} \mathrm{s}{ }^{-1} \mathrm{~m}^{-2}$ en el mes de abril.

En la figura 1 se puede observar que existen dos tendencias en el comportamiento de la radiación en ambos transectos. La primera se encuentra comprendida entre los meses de febrero a septiembre y la segunda de octubre a enero del siguiente año. En la primera se observa que en ambos transectos existe mayor cantidad de radiación en el mes de abril y que esta radiación tiende a disminuir hasta el mes de julio del mismo año y luego aumenta en ambos casos en el mes de agosto y septiembre. En la segunda etapa la cantidad de radiación es mucho menor en ambos transectos. Los valores bajos de radiación de octubre a enero en los sitios no quemados se deben principalmente a que la altura de las plantas herbáceas era mayor que la altura de los brinzales y por lógica, esta misma altura impedía que la radiación llegará al piso forestal en grandes cantidades.

La posición de los sitios de muestreo a lo largo de la pendiente dentro de cada transecto no influyó de manera directa en la cantidad de radiación que llegó al piso forestal en las dos condiciones evaluadas y también a lo largo del período de estudio las diferencias no fueron significativas en la cantidad de radiación en los sitios dentro de cada uno de los transectos.

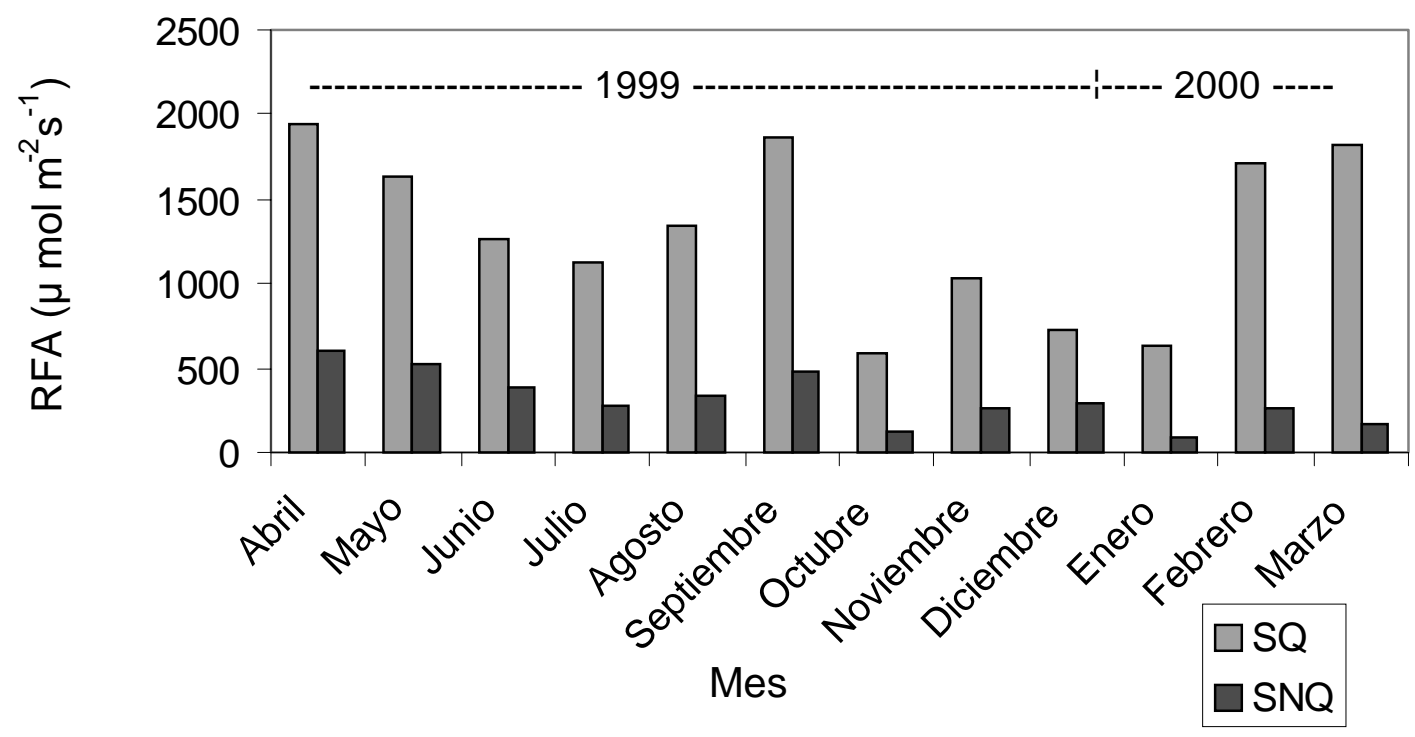

Figura 1. Radiación fotosintéticamente activa a través del tiempo de evaluación en los sitios quemados y no quemados. 


\section{Propiedades físico-químicas de los suelos}

Diferencias entre transectos al inicio de la estación de crecimiento. En la evaluación realizada once meses después del incendio, los resultados de las propiedades físico-químicas del suelo se dividieron en dos grupos. En el primer grupo se incluyeron las propiedades que no se modificaron en forma significativa con el fuego. En este grupo se encuentra la textura del suelo, la capacidad de intercambio catiónico, el pH, el fósforo y el calcio (Tabla 1). En el segundo grupo se incluyeron las propiedades en las que se observó un efecto significativo del fuego, como la materia orgánica, nitrógeno inorgánico, potasio, magnesio, hierro, cobre, zinc y manganeso (Tabla 1).

Es importante observar que en todos los casos en que hubo un efecto significativo del fuego, el efecto fue el de reducir la concentración de los nutrimentos, con respecto a los valores observados en el transecto no quemado. Sin embargo, esta reducción no fue similar en todas las propiedades evaluadas. Por ejemplo, el nitrógeno inorgánico se redujo solo en un $22 \%$ con respecto al transecto no quemado, mientras que el potasio se redujo en más del $85 \%$. El resto de los nutrimentos que fueron afectados por el incendio se redujeron en alrededor de un $40 \%$ a $50 \%$ (Tabla 1 ).

Tabla 1. Valores promedio de diferentes propiedades físico-químicas del suelo en los transectos no quemado (TNQ) y quemado (TQ) al inicio de la estación de crecimiento (Inicio), once meses después del incendio y al final de la estación de crecimiento (Final), veinte meses después del incendio

\begin{tabular}{|c|c|c|c|c|}
\hline PROPIEDAD & $\begin{array}{c}\text { TNQ } \\
\text { (Inicio) }\end{array}$ & $\begin{array}{c}\text { TNQ } \\
\text { (Final) }\end{array}$ & $\begin{array}{c}\mathrm{TQ} \\
\text { (inicio) }\end{array}$ & $\begin{array}{c}\mathrm{TQ} \\
\text { (Final) }\end{array}$ \\
\hline $\mathrm{pH}$ & $5,38 \mathrm{a}$ & $5,45 a$ & $5,04 a$ & $4,65 a$ \\
\hline Materia orgánica (MO \%) & $14,74 a$ & $15,97 a$ & $7,27 \mathrm{~b}$ & $7,40 \mathrm{~b}$ \\
\hline Nitrógeno inorgánico (NIN mg kg$\left.{ }^{-1}\right)$ & $8,05 a$ & $7,18 a$ & $7,14 a$ & $7,61 a$ \\
\hline Fósforo ( $\left.\mathrm{P} \mathrm{mg} \mathrm{kg}{ }^{-1}\right)$ & $9,06 \mathrm{a}$ & $7,93 a$ & $21,00 a$ & $16,00 \mathrm{a}$ \\
\hline Potasio $\left(\mathrm{K} \mathrm{mg} \mathrm{kg}^{-1}\right)$ & $254,85 a$ & $242,57 a$ & $32,28 b$ & $112,42 b$ \\
\hline Calcio (Ca mg kg ${ }^{-1}$ ) & $418,70 a$ & $396,00 a$ & $415,28 a$ & $281,86 a$ \\
\hline Magnesio $\left(\mathrm{Mg} \mathrm{mg} \mathrm{kg}^{-1}\right)$ & $119,78 a$ & $127,00 \mathrm{a}$ & $0,32 b$ & $8,73 b$ \\
\hline Hierro (Fe mg kg $\left.{ }^{-1}\right)$ & $97,74 a$ & $97,22 a$ & $40,58 \mathrm{~b}$ & $39,70 \mathrm{~b}$ \\
\hline Cobre (Cu mg kg $\left.{ }^{-1}\right)$ & $1,35 a$ & $0,96 \mathrm{a}$ & $0,76 \mathrm{~b}$ & $0,53 a$ \\
\hline Zinc $\left(\mathrm{Zn} \mathrm{mg} \mathrm{kg}{ }^{-1}\right)$ & $1,12 \mathrm{a}$ & $0,95 a$ & $0,75 b$ & $0,50 a$ \\
\hline Manganeso ( $\mathrm{Mn} \mathrm{mg} \mathrm{kg}^{-1}$ ) & $12,25 a$ & $8,44 a$ & $8,08 \mathrm{~b}$ & $4,05 b$ \\
\hline $\begin{array}{l}\text { Capacidad de intercambio catiónico (CIC } \\
\mathrm{cmol}_{(+)} \mathrm{kg}^{-1} \text { ) }\end{array}$ & $40,00 a$ & $33,00 a$ & $20,00 a$ & $23,00 a$ \\
\hline Arena (\%) & $38,00 a$ & $38,00 a$ & $38,00 a$ & $40,00 a$ \\
\hline Limo (\%) & $44,00 \mathrm{a}$ & $44,00 \mathrm{a}$ & $44,00 \mathrm{a}$ & $40,00 \mathrm{a}$ \\
\hline Arcilla (\%) & $18,00 \mathrm{a}$ & $18,00 a$ & $18,00 a$ & $20,00 a$ \\
\hline
\end{tabular}


Dinámica estacional en los dos transectos. Al comparar los valores promedio obtenidos al inicio de la época de lluvias, seis meses después de la evaluación inicial, se observó que en el transecto quemado existen propiedades químicas que aumentaron en concentración, como es el caso del potasio y el magnesio, en más de 3 y 27 veces, respectivamente. En cambio, el calcio y el manganeso tendieron a disminuir con el tiempo en un $32 \%$ y $50 \%$, respectivamente, hacia la evaluación final. Por otro lado, el resto de los nutrimentos y propiedades del suelo fueron relativamente estables a través del período de evaluación en el transecto quemado, incluyendo la materia orgánica, nitrógeno inorgánico, fósforo, hierro, cobre, zinc, capacidad de Intercambio catiónico y la textura (Tabla 1).

Por otro lado, en el transecto no quemado se observa que existen nutrimentos que disminuyeron en concentración ligeramente con el tiempo (entre un $10 \%$ y $30 \%$ ), como es el caso del nitrógeno inorgánico, fósforo, cobre, zinc y el manganeso. Sin embargo, al igual que en el transecto quemado, se encontró un grupo de variables cuya concentración fue relativamente estable a través del período de evaluación, como es el caso de la materia orgánica, potasio, calcio, magnesio, hierro, capacidad de Intercambio catiónico y la textura del suelo.

Debido a las diferencias en la dinámica estacional seguida por algunas propiedades del suelo en los dos transectos, en la evaluación realizada al final del período de estudio, a los 20 meses de ocurrido el incendio y a siete meses después de la primera evaluación, se encontró que los transectos aún presentan diferencias en la concentración de algunas características, como la materia orgánica, potasio, magnesio, hierro y manganeso (Tabla 1). Es decir, a pesar de los incrementos ocurridos en estos elementos en el transecto quemado, éstos no fueron significativos para compensar la pérdida inicial ocasionada por el incendio. En el caso del $\mathrm{pH}$, ocurre un fenómeno inverso, pues los valores en el sitio quemado tienden a reducirse con el tiempo, produciendo un efecto de acidificación del suelo.

Comportamiento espacial dentro de los transectos. Al inicio del estudio se encontró que el incendio también modificó el patrón espacial de algunas propiedades de los suelos, con respecto al patrón original existente en el transecto no quemado. Por ejemplo, en el transecto no quemado, el hierro y zinc tienden a aumentar su concentración de los sitios de menor a mayor elevación y el manganeso y cobre tienden a reducir su concentración hacia los sitios más elevados. Mientras que en el transecto quemado, estos elementos no siguen un patrón espacial definido, con una concentración relativamente uniforme a lo largo del transecto (hierro y manganeso) o con una variación elevada pero con un patrón espacial aleatorio (zinc y cobre) (Fig. 2). Sin embargo, con excepción de los elementos anteriores, en el resto de las propiedades evaluadas no se observó un cambio en el patrón espacial como resultado del incendio. Así por ejemplo, el potasio muestra la misma tendencia a disminuir su concentración en los sitios de mayor elevación en ambos transectos. Por otro lado, el $\mathrm{pH}$, materia orgánica, nitrógeno inorgánico y magnesio varían en forma aleatoria, sin seguir un patrón espacial definido en ninguno de los dos transectos (Fig. 3).

Con excepción del zinc y materia orgánica, que con el tiempo modificaron su patrón espacial inicial en transecto no quemado, en el resto de los elementos y propiedades, el patrón espacial se mantiene relativamente estable en el transecto no quemado hasta la última evaluación realizada en enero de 2000. En cambio, en el transecto quemado parece ser mucho más dinámico, pues al menos cuatro de los elementos y propiedades que al principio no mostraban un patrón espacial definido (hierro, zinc, manganeso y materia orgánica), en la 
última evaluación mostraron una clara tendencia espacial a disminuir hacia las partes más elevadas del transecto los tres primeros y a incrementar en la misma dirección el último (Fig. 4), con valores de $R^{2}$ superiores a 0,66 . Los otros nutrimentos y propiedades, por ejemplo el cobre, potasio, $\mathrm{pH}$, nitrógeno inorgánico y magnesio, no modificaron su patrón espacial original en el transecto quemado (Fig. 5).

Debido a la diferente dinámica observada en los dos transectos, en la evaluación final se presenta una situación diferente en cuanto al patrón espacial de algunos elementos en los dos transectos. Por ejemplo, en algunos elementos como el manganeso, la diferente dinámica existente en los transectos ocasionó que las diferencias iniciales en el patrón espacial se eliminaran con el tiempo; es decir, en la evaluación final el patrón espacial del manganeso en el transecto quemado es similar al patrón existente en el transecto no quemado (Fig. 4). Sin embargo, a pesar de la dinámica particular observada en cada uno de los transectos, en la mayoría de las propiedades evaluadas, las diferencias iniciales en el patrón espacial entre los dos transectos se mantiene hasta la última evaluación realizada.

\section{DISCUSIÓN}

\section{Radiación fotosintéticamente activa}

Los incendios provocan que la vegetación competidora sea eliminada en primera instancia, incrementando la cantidad de radiación que llega al piso forestal, favoreciendo a la repoblación del claro ocasionado por el disturbio. A este respecto, Jobidon (1994) y Ángeles et al., (1997) indican que la cobertura de malezas reduce dramáticamente la cantidad de luz a nivel del suelo, ocasionando una menor oportunidad de los brinzales de Picea mariana y Pinus patula, respectivamente, para abastecerse de este recurso. Esta situación se corrige realizando un control manual de la vegetación competidora. Este efecto es similar al ocasionado por los incendios que eliminan a todo tipo de vegetación competidora, promoviendo la germinación y establecimiento de la especie de interés. Estas especies pueden desarrollarse y ocupan un espacio de crecimiento rápidamente porque existe disponibilidad de factores de crecimiento.

En el caso del presente estudio, se detectó que en los sitios no quemados la presencia de la vegetación herbácea redujo drásticamente la cantidad de luz que llega al piso forestal, sobre todo, en el otoño y en el invierno. Para el caso de los sitios quemados, la liberación de la vegetación herbácea, permitió que se incrementara la cantidad de luz que llega al piso forestal de manera sustancial, inclusive a pesar de la presencia de nubes y neblina. Estos incrementos de luz pueden persistir a través del tiempo como lo han señalado Ángeles et al. (1997).

Se ha señalado que existen diferencias en la cantidad de luz recibida en función de la ubicación de los sitios conforme a la posición en la pendiente en un estudio realizado en Pinus strobus en los montes Apalaches (Elliott y Vose, 1993). En este estudio, los valores de radiación oscilaron entre $800 \mu \mathrm{mol} \mathrm{s}{ }^{-1} \mathrm{~m}^{-2} \mathrm{y}$ $1100 \mu \mathrm{mol} \mathrm{s}^{-1} \mathrm{~m}^{-2}$ en los sitios con exposición Oeste y de $1100 \mu \mathrm{mol} \mathrm{s}{ }^{-1} \mathrm{~m}^{-2} \mathrm{a}$ $1400 \mu \mathrm{mol} \mathrm{s}^{-1} \mathrm{~m}^{-2}$ en los sitios con exposición Sur. En el caso del presente estudio, en donde la exposición de los transectos es hacia el norte, la posición de los sitios a lo largo de la pendiente no influyó sobre la cantidad de radiación que llegó al piso forestal. 


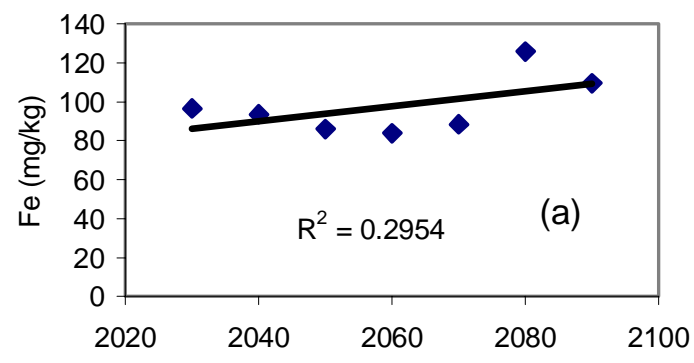

Altura (msnm) de los sitios de muestreo

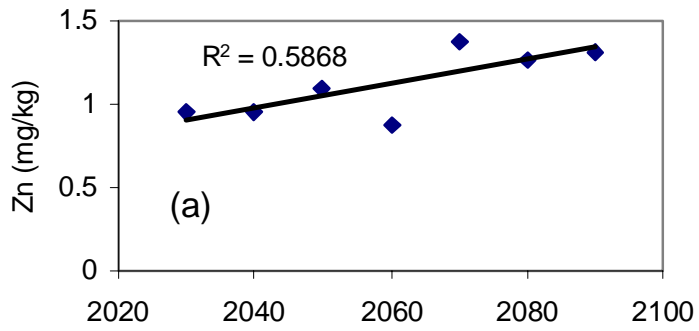

Altura (msnm) de los sitios de muestreo

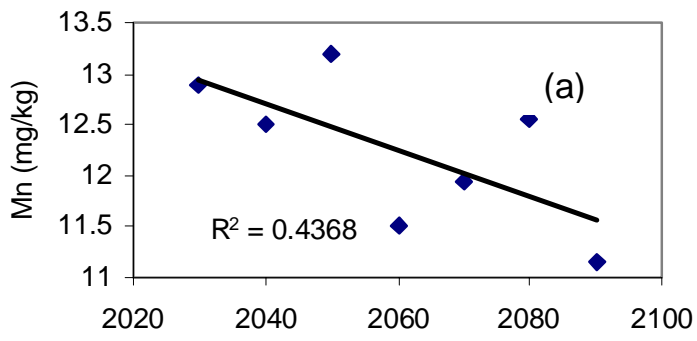

Altura (msnm) de los sitios de muestreo

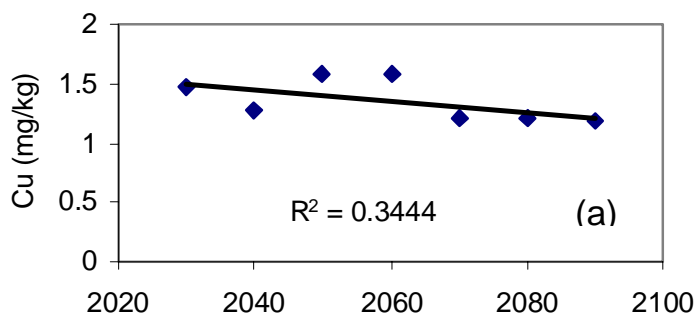

Altura (msnm) de los sitios de muestreo
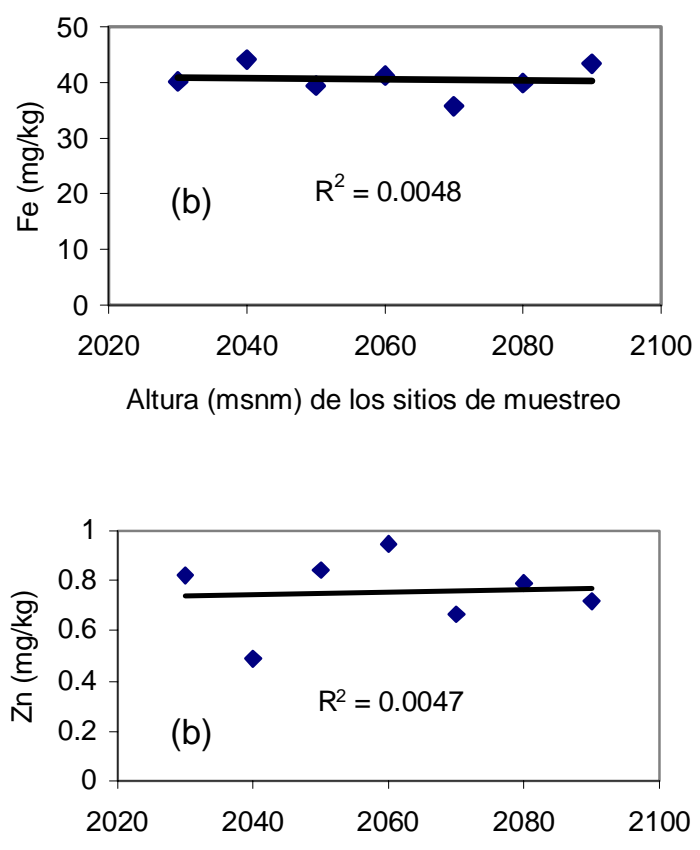

Altura (msnm) de los sitios de muestreo

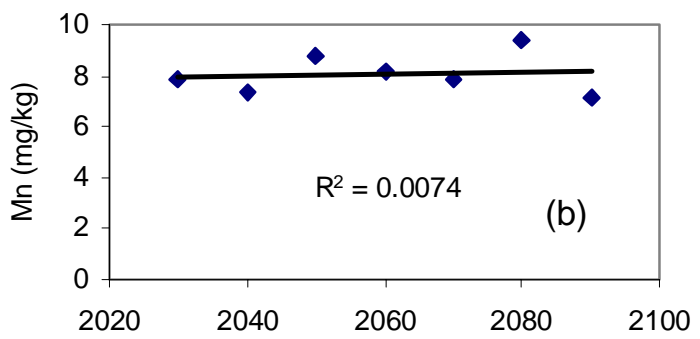

Altura (msnm) de los sitios de muestreo

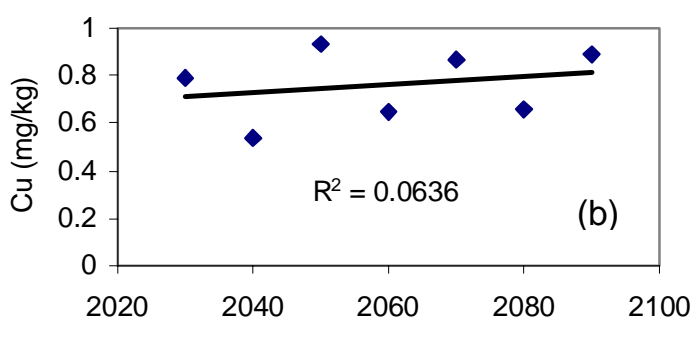

Altura (msnm) de los sitios de muestreo

Figura 2. Tendencia del hierro, zinc, manganeso y cobre $\left(\mathrm{mg} \mathrm{kg}^{-1}\right)$ al inicio de la estación de crecimiento en el transecto no quemado (a) y quemado (b). 

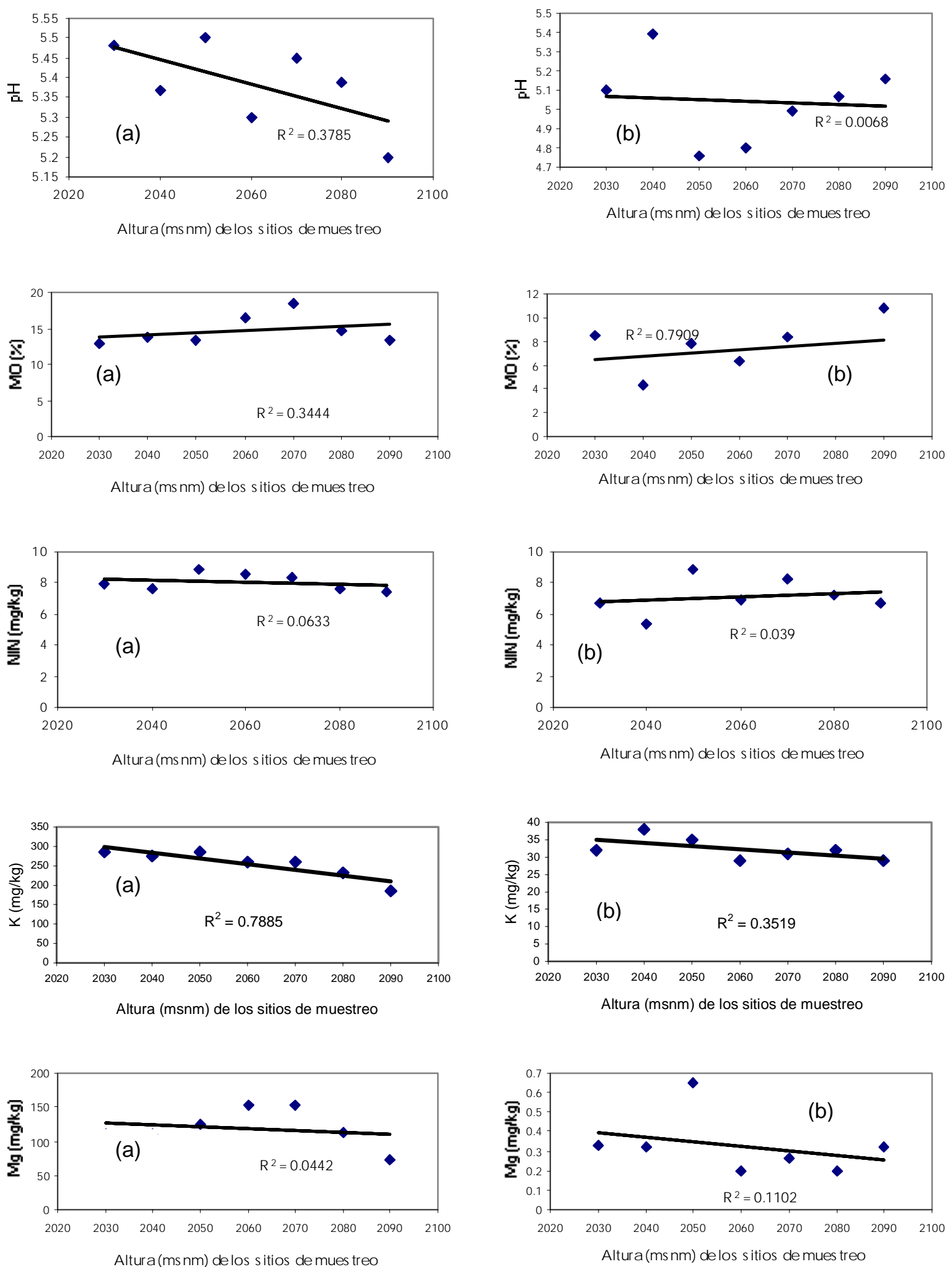

Figura 3. Tendencia del pH, la materia orgánica (\%), nitrógeno inorgánico, potasio, y magnesio $\left(\mathrm{mg} \mathrm{kg}^{-1}\right)$ al inicio de la estación de crecimiento en el transecto no quemado (a) y quemado (b). 


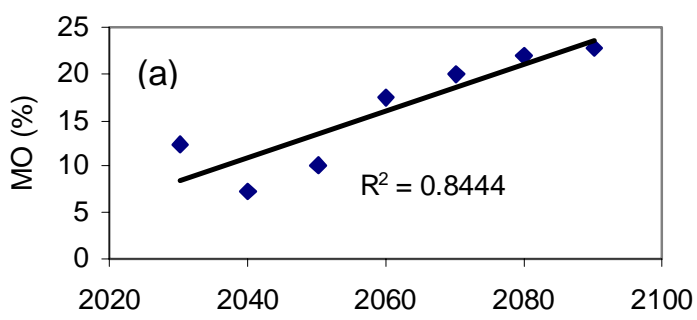

Altura (msnm) de los sitios de muestreo

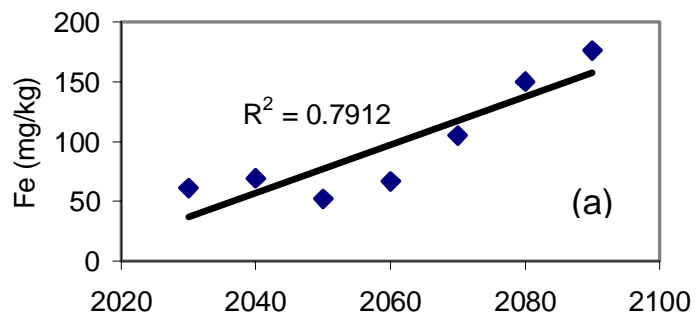

Altura (msnm) de los sitios de muestreo

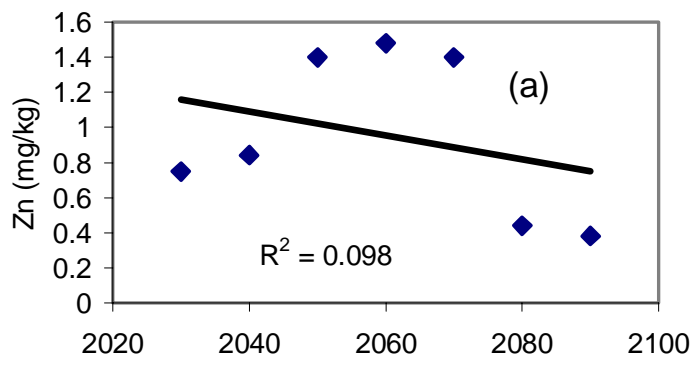

Altura (msnm) de los sitios de muestreo

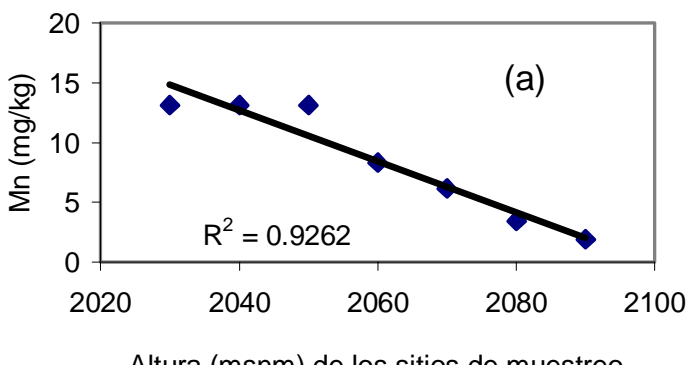

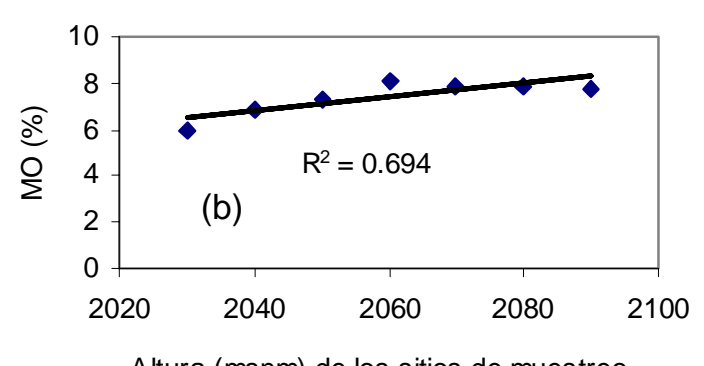

Altura (msnm) de los sitios de muestreo
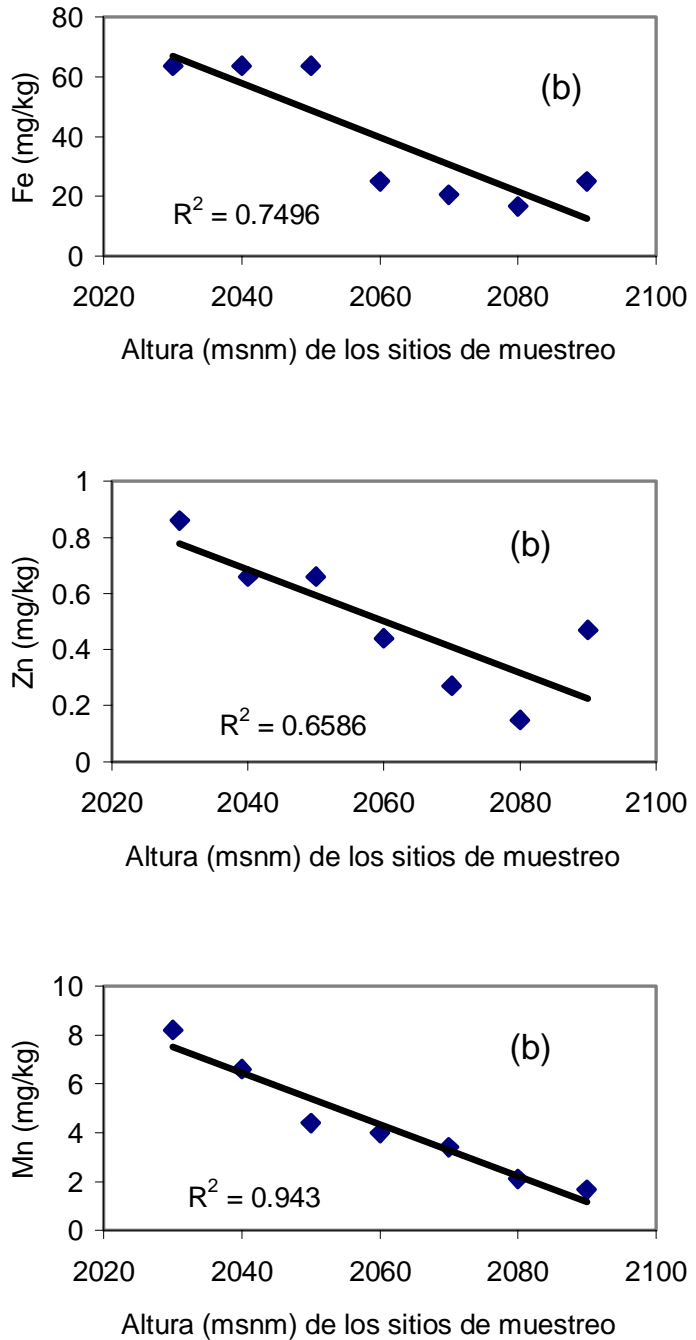

Figura 4. Tendencia de la materia orgánica (\%), del hierro, zinc, manganeso $\left(\mathrm{mg} \mathrm{kg}^{-1}\right)$ al final de la estación de crecimiento en el transecto no quemado (a) y quemado (b). 

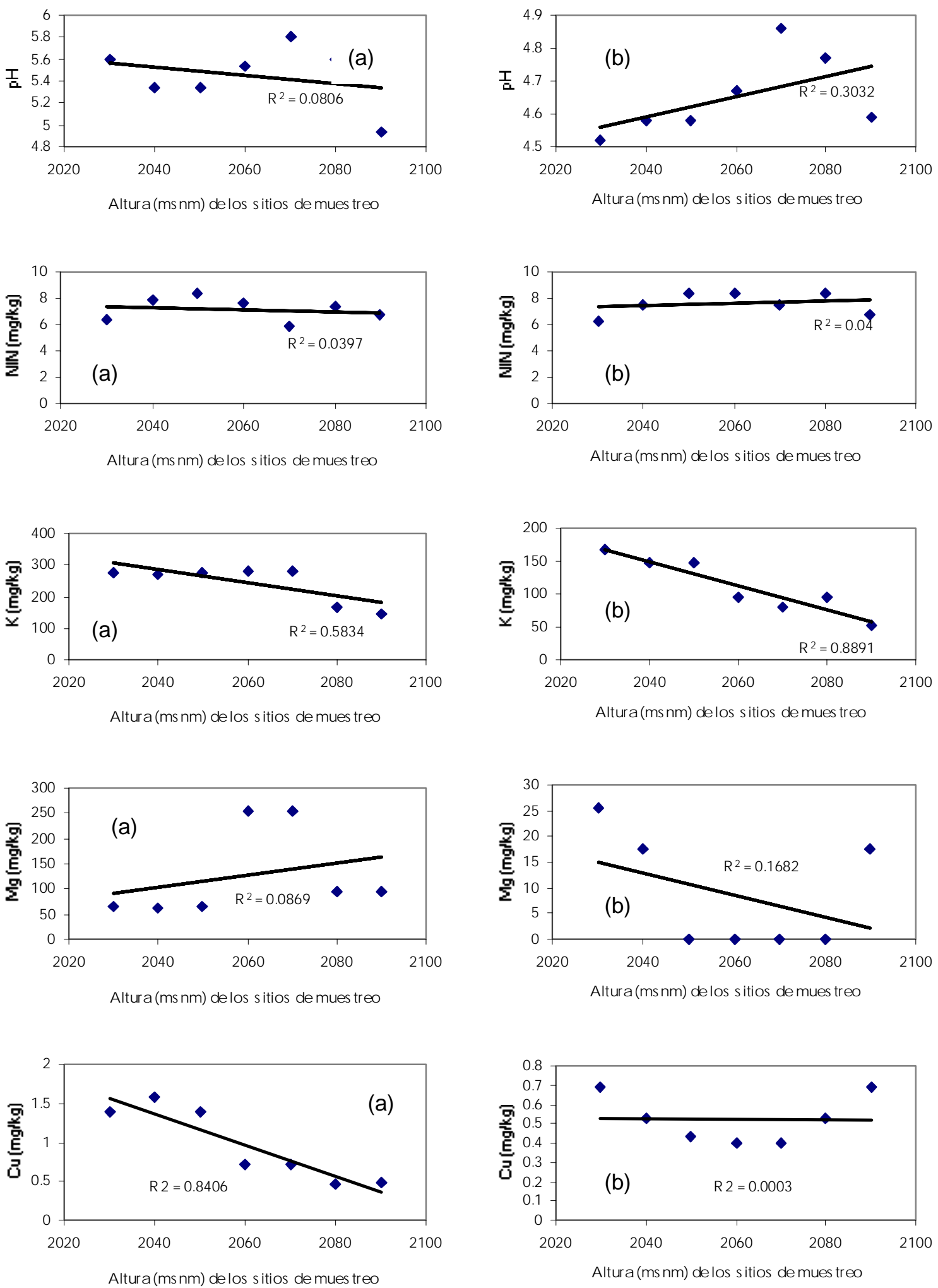

Figura 5. Tendencia del $\mathrm{pH}$, nitrógeno inorgánico, potasio, magnesio y cobre $\left(\mathrm{mg} \mathrm{kg}^{-1}\right)$ al final de la estación de crecimiento en el transecto no quemado (a) y quemado (b). 


\section{Propiedades físico-químicas de los suelos}

Se sabe que existen cambios en las propiedades químicas del suelo después de que se presenta un incendio. Autores como Viro (1974) indican que existen incrementos de algunos nutrimentos después de un incendio, pero algunos otros autores reportan lo contrario, como es el caso de Brais et al. (1995) quienes indican que existe una disminución del $\mathrm{pH}$ (de 5,5 a 3,5) de la capacidad de intercambio catiónico (de $72,5 \mathrm{cmol}(+) \mathrm{kg}^{-1}$ a $\left.39,6 \mathrm{cmol}(+) \mathrm{kg}^{-1}\right)$, y de la concentración de calcio, magnesio, potasio y fósforo en los suelos boreales de Québec, una vez que se ha presentado un incendio. En el presente estudio también se observó un ligero cambio en $\mathrm{pH}$ y en la capacidad de intercambio catiónico, pero estas diferencias no fueron significativas estadísticamente. Sin embargo, en las otras propiedades químicas, como la materia orgánica, nitrógeno inorgánico, potasio, magnesio, zinc, cobre, hierro y manganeso, sí se encontró una reducción significativa, de entre $20 \%$ y el $60 \%$ con respecto a los sitios no quemados.

Fassbender y Bornemisza (1987), indican que el incendio repercute especialmente en la reducción de la materia orgánica y en los elementos nutritivos, entre otros, incidiendo así sobre la fertilidad del suelo, como ha sucedido en este caso en donde, como se ha señalado en el párrafo anterior, la cantidad de materia orgánica disminuye en más del $50 \%$.

A este respecto, Kozlowski y Palardy (1997) mencionan que la alta disponibilidad de nutrimentos después de un incendio puede o no mejorar la calidad del sitio. Si los minerales se lixivian por debajo de la zona radical o son lavados de la superficie, la calidad del sitio será menor. Sin embargo, si los minerales solubles no son lixiviados en la zona radical y son absorbidos por las raíces, la calidad del sitio se mejorará.
Además, las cenizas que quedan en el campo (Fassbender y Bornemisza, 1987), tienen una composición química que es alta en elementos nutritivos. Estos elementos, dependiendo de su solubilidad se pierden rápidamente por lixiviación, erosión y drenaje, como es el caso del nitrógeno en forma de $\mathrm{NO}_{3^{-}}$y $\mathrm{NH}_{4+}$, que se presentan en la solución del suelo y que percolan por gravedad.

En el presente estudio, se ha observado que la cantidad de nutrimentos en el suelo después de once meses de ocurrido el incendio, fue menor en algunos casos, como en el potasio, magnesio, hierro, cobre, zinc y manganeso. Esta situación puede deberse a varias causas, por ejemplo, el tiempo que ha transcurrido después del incendio es suficiente para que existan pérdidas por erosión, lixiviación 0 drenaje. Por otro lado, también es posible que en este lapso, las plantas hayan absorbido los nutrimentos liberados por el incendio. Este es un aspecto que se analizará posteriormente al evaluar la concentración de nutrimentos presentes en los brinzales en las dos condiciones del sitio (quemado y no quemado).

En un estudio reciente, Zabowski (2000) encontró que el $\mathrm{N}$ se redujo hasta en un $46 \%$ en los sitios quemados de un bosque de Pinus ponderosa y Pseudtsuga menziesii. En cambio, en el presente estudio, se encontró un resultado diferente, ya que desde la primera evaluación, once meses después del incendio, no se encontraron diferencias significativas en la concentración de este nutrimento entre las dos condiciones de estudio. Es posible que en el período posterior al incendio, previo a la fecha de la primera evaluación ya se haya recuperado la concentración en los suelos de manera rápida. A este respecto, Kozlowski y Pallardy (1997) mencionan que a pesar de las grandes pérdidas de nitrógeno por combustión, este nutrimento es rápidamente adicionado al suelo por la precipitación, por el incremento en la actividad de los microorganismos y por fijación del mismo. 
Rodríguez (1996) señala que el fósforo es un elemento que generalmente se incrementa después de un incendio. Aún cuando en el presente estudio no se encontraron diferencias significativas en este elemento, sí se observó una tendencia a aumentar en los sitios quemados. Fassbender y Bornemisza (1987) también indican que el $P$ es un elemento relativamente estable en los suelos y mencionan que no presenta compuestos inorgánicos, como los nitrogenados que pueden ser volatilizados y altamente lixiviados. Esta gran estabilidad resulta de una baja solubilidad.

En el presente estudio se encontró que al final del período de evaluación la materia orgánica, potasio y magnesio tienden a incrementar su concentración con respecto a los valores iniciales en los sitios quemados, principalmente por los aportes del bosque circundante, por los residuos de las efímeras, por los propios brinzales establecidos en el transecto y por los lavados del aire y de la vegetación en general (Duvigneaud y Denaeyer-De Smet, 1970).

En el mismo sentido, Waring y Schlesinger (1985) y Trinidad (1998) indican que la neblina proveniente del océano posee generalmente grandes concentraciones de iones como el potasio, magnesio, calcio y sodio, situación que favorece a un gran número de comunidades forestales; además, señalan que el intemperismo de la roca es un factor fuente de nutrimentos a los ecosistemas forestales, ya que los iones solubles son liberados y se encuentran disponibles para ser aprovechados por las plantas y que, de hecho, del $80 \%$ al $100 \%$ de los aportes de calcio, magnesio, potasio y fósforo se derivan del intemperismo de la roca que en este caso es de origen ígneo y cuyos minerales primarios son silicatos con diversos cationes entre los que se encuentran el aluminio, calcio, sodio, potasio y magnesio y algunos elementos en menor proporción como son hierro y manganeso.
En los sitios no quemados el comportamiento es prácticamente el mismo a través del tiempo de evaluación. A este respecto, Perry (1994) menciona que la materia orgánica tiende a incrementar su cantidad a través del tiempo, como lo reportado por Homann et al., (1994) quienes señalan que después de tres años la mayoría de los nutrimentos tienden a estabilizar su concentración en el suelo, pero que este tiempo es variable dependiendo de la reducción y el cambio en la calidad de la hojarasca y materia orgánica que se adiciona al suelo después de un disturbio. Como puede observarse en el presente estudio a siete meses de la primera evaluación, los nutrimentos en el suelo (magnesio, potasio, calcio) presentan esta tendencia a estabilizarse.

Brown y Davis (1973), Perry (1994) y Rodríguez (1996) indican que uno de los efectos químicos más inmediatos del incendio, es el de liberar los elementos minerales que se lixivian en el suelo por ejemplo: fósforo, calcio, hierro y potasio que se vuelven más abundantes y estimulan el crecimiento de las plantas. Sin embargo, las condiciones de relieve pueden hacer que estos elementos se pierdan por erosión y sean desplazados a las partes bajas de las cuencas, de los ríos y lagos causando efectos tóxicos por su acumulación. En el estudio se encontró que el potasio presenta esta tendencia y que el hierro tiende a acumularse hacia las partes altas, aunque esta acumulación no es realmente significativa.

Por otro lado, Binkley (1986), indica que la pendiente del suelo influye sobre la lixiviación, la erosión, la distribución de nutrimentos y la cubierta vegetal, como fue en el caso del presente estudio. En los sitios quemados ningún nutrimento presentó un patrón espacial definido con respecto a la posición del sitio en la pendiente al inicio del estudio; sin embargo, al final del estudio, el hierro, zinc, manganeso y materia orgánica presentaron un patrón bien definido de distribución a lo largo del transecto, 
tendiendo a disminuir su concentración hacia las partes más elevadas en los tres primeros y a aumentar en la misma dirección en el caso de la materia orgánica.

\section{CONCLUSIONES}

El incendio aumentó la cantidad de radiación fotosintéticamente activa que llegó al piso forestal a lo largo de todo el año. Las diferencias en radiación a través del tiempo de evaluación se mantuvieron constantes, sobre todo en los sitios quemados, debido principalmente a los espacios que no han sido cubiertos por la vegetación. Por otro lado, la cantidad de radiación fotosintéticamente activa que llegó al piso forestal también fue homogénea a lo largo de la pendiente en ambos transectos.

El incendio produjo una disminución en la concentración de materia orgánica, potasio, magnesio, hierro, cobre, zinc y manganeso. Estacionalmente, la concentración de materia orgánica, nitrógeno inorgánico y fósforo se incrementaron en mayor cantidad en los sitios quemados, al grado que después de siete meses ya habían recuperado las concentraciones existentes en los sitios no quemados. El incendio también modificó el patrón de distribución espacial del hierro, zinc, manganeso y la capacidad de intercambio de cationes, con respecto al que existía en la primera evaluación. Así mismo, hacia el final de la evaluación el hierro, zinc y manganeso en los sitios quemados, tendieron a disminuir hacia las partes altas del terreno, mientras que la materia orgánica tendió a aumentar en la misma dirección.

\section{REFERENCIAS}

Alcántara, G.G. 1992. Análisis vegetal. In: G.G. Alcántara; J.D. Etchevers Barra y A. Aguilar, eds. Los análisis físicos y químicos, su aplicación en agronomía. Centro de Edafología. Colegio de
Postgraduados, Montecillo. México. p:108-124.

Ángeles, G.; A. Velázquez; J. Vargas; J. Velásquez y M. Musalem. 1997. El control de malezas y la disponibilidad de luz y agua durante el establecimiento de un rodal natural de Pinus patula, en México. Invest. Agr. Sist. Recur. For. 6 (1 y 2):133-145.

Attiwill, P.M. y M.A. Adams. 1993. Nutrient cycling in forests. New Phytol. 124: 561-582.

Binkley, D. $1986 . \quad$ Forest nutrition management. John Wiley and Sons. Nueva York. 339 p.

Brais, S.; C. Camiré; Y. Bergeron y D. Paré. 1995. Changes in nutrient availability and forest floor characteristics in relation to stand age and forest composition in the southern part of the boreal forest of northwestern Quebec. For. Ecol. Man. 76:181-189.

Brown, A.A. y K.P. Davis. 1973. Forest fire, control and use. McGraw-Hill (Ed). New York. 686 p.

Canham, Ch.D.; J.S. Denslow; W.J. Platt; J.R. Runkle; T.A. Spies y P.S. White. 1990. Light regimes beneath closed canopies and tree-fall gaps in temperate and tropical forests. Can. J. For. Res. 20:620-631.

Dai, X. 1996. Influence of light conditions in canopy gaps on forest regeneration: a new gap light index and its application in a boreal forest in eastcentral Sweden. For. Ecol. Man. 84:187-197.

Duvigneaud, P. y S. Denaeyer-De Smet. 1970. Biological cycling of minerals in temperate deciduous forests. In: Reichle, D.E., ed. Analysis of temperate forest ecosystems, Ecological Studies 1. Springer-Verlag, N.Y. 463 p. 
Eibl, B.; Ch. Woodward y F. Szczipanski. 1998. Natural regeneration under two systems of selective cutting and undisturbed forest in Misiones Argentina. http://iufro.boku.ac.at/ iufronet/d1/wu10700/unpub/montag95 .htm.

Elliot, K.J. y J.M. Vose. 1993. Site preparation burning to improve southern Appalachian pine-hardwood stands: photosynthesis, water relations, and growth of planted Pinus strobus during establishment. Can. J. For. Res. 23:2278-2285

Etchevers B., J.D.; C.A. Ortiz y J.L. Tovar. 1992. Análisis de suelos. In: G.G. Alcántara; J.D. Etchevers B. y A. Aguilar, eds. Los análisis físicos y químicos, su aplicación en agronomía. Centro de Edafología. Colegio de Postgraduados. Montecillo. México. p:78-81.

Fassbender, H.W. y E. Bornemiza. 1987. Química de suelos con énfasis en suelos de América Latina. IICA, Costa Rica. 420 p.

García, E. 1988. Modificaciones al sistema climático de Köppen para adaptarlo a las condiciones de la República Mexicana. UNAM. México. 153 p.

Gray, A.N. y T.A. Spies. 1997. Microsite controls on tree seedling establishment in conifer forest canopy gaps. Ecology 78(8):2458-2473.

Homann, P.S.; D.W. Cole; H.V. Miegroet y J.E. Compton. 1994. Relationship between cation and nitrate concentrations in soil solutions from mature and harvested red alder stands. Can. J. For. Res. 24:1646-1652

INEGI. 1996. Cuaderno estadístico municipal Zacualtipán de Ángeles estado de Hidalgo. INEGI, México. $360 \mathrm{p}$.
Jobidon, R. 1994. Light threshold for optimal black spruce (Picea mariana) seedling growth and development under brush competition. Can. J. For. Res. 24:1629-1635.

Knoepp, J.D. y W.T. Swank. 1993. Site preparation burning to improve southern Appalachian pine-hardwood stands: nitrogen responses in soil, soil water, and streams. Can. J. For. Res. 23:2263-2270

Kozlowski, T.T.; P.J. Kramer y S. G. Pallardy. 1991. The physiological ecology of woody plants. Academic Press. San Diego, Cal. 657 p.

Kozlowski, T.T. y S.G. Pallardy. 1997. Growth control in woody plants. Academic Press. San Diego, Cal. $641 \mathrm{p}$.

Landsberg, J.J. y S.T. Gower. 1997. Applications of physiological ecology to forest management. Academic Press. San Diego, Cal. 354 p.

Olvera, V.M.; S. Moreno; B. Figueroa. 1996. Sitios permanentes para la investigación silvícola, manual para su establecimiento. Universidad de Guadalajara, Jalisco. México. p:11-13.

Peck, T.R. y S. W. Melsted. 1973. Field sampling for soil testing. In: Walsh y Beaton, eds. Soil Testing and Plant Analysis. Wisconsin. p:67-75.

Perry, D.A. 1994. Forest ecosystems. The Johns Hopkins University Press Ltd. Londres. p:379-380.

Poulson, T.L. y W.J. Platt. 1989. Gap light regimes influence canopy tree diversity. Ecology 70 (3):553-555

Pritchett, L.W. 1986. Suelos forestales, propiedades, conservación y mejoramiento. Ed. Limusa. México. 638 p. 
Rodríguez, T. D.A. 1996. Incendios forestales. Ed. UACh-Mundi Prensa. México. $630 \mathrm{p}$.

SAS. 1997. SAS/STAT guide for personal computers, version $6^{\text {th }}$. SAS Institute Inc., Cary NC. 584 p.

Sipe, T.W. y F.A. Bazzaz. 1995. Gap partitioning among maples (Acer) in central New England: survival and growth. Ecology 76: 1587-1602.

Smith, D.M.; B.C. Larson; M.J. Kelty y P.M.S. Ashton. 1997. The practice of silviculture: applied forest ecology. 9a. ed. Ed. John Wiley and Sons. Nueva York. $537 \mathrm{p}$.

Spurr, S.H. y B.V. Barnes. 1980. Forest ecology. 2a. ed. Ronald Press. Nueva York. $571 \mathrm{p}$.

Stein, W.I. 1992. Regeneration surveys and evaluation. In: Hobbs, S.D.; S.D. Tesch; P.W. Owston; R.E. Stewart; J.C. Tappeiner y G.E. Wells, eds. Reforestation practices in South Western Oregon and Northern California. For. Res. Lab. Oregon State University. Corvallis, Oregon. p:347-378.

Stewart, G.H.; A.B. Rose y T.T. Veblen. 1991. Forest development in canopy gaps in old-growth beech (Nothofagus) forests. New Zealand J. Veg. Sci. 2:679-690.
Swift, LI.W.; K.J. Elliott.; R.D. Ottmar y R.E. Vihnanek. 1993. Site preparation burning to improve southern Appalachian pine-hardwood stands: fire characteristics and soil erosion, moisture, and temperature. Can. J. For. Res. 23:2242-2254

Tan, K.H. 1996. Soil sampling, preparation and analysis. Mercel Dekker, Inc. Nueva York. 408 p.

Trinidad, S.A. 1998. Apuntes de asignatura de Edafología Forestal. Instituto de Recursos Naturales. Colegio de Postgraduados. Montecillo, Texcoco, México. (inédito).

USDA. 1996. Natural technical report of forest health. FS605. Forest Service. http://willow.ncfes.umn.edu/fhm/pubs/ 96scons.htm

Viro, P.J. 1974. Effects of forest fire on soil. In. Kozlowski, T.T. y E. Ahlgren, eds. Fire and ecosystems. Academic Press. $354 \mathrm{p}$.

Waring, R.H. y W.H. Schlesinger. 1985. Forest ecosystems, concepts and management. Academic Press, Inc. Orlando, Florida. pp:40-43.

Zabowski, I. 2000. Wildfire Effects on Soil Nutrients. http://www.cfr.washington. edu/Faculty/faculty_reports/zabowski2. $\mathrm{htm}$.

1 Centro de Investigaciones Forestales-UAEH. Tulancingo, Hidalgo.

2 Especialidad Forestal. Colegio de Postgraduados. km 35.5 Carretera México-Texcoco. Montecillo 56230 Edo. de México.

3 Programa de Edafología. Colegio de Postgraduados. km 35.5 Carretera México-Texcoco. Montecillo 56230 Edo. de México.

4 División de Ciencias Forestales. Universidad Autónoma de Chapingo. Apdo Postal 112. Chapingo 56230 Edo de México. c.e. mmusalem@www.citsatex.com.m*.

5 Campo Agrícola Experimental Valle de México. INIFAP. Chapingo, Edo. de México.

Manuscrito recibido el 11 de marzo de 2002.

Aceptado el 24 de junio de 2002.

Este documento se debe citar como:

Maycotte M., C.C.; A. Velázquez M.; J.J. Vargas H.; A. Trinidad S.; M.A. Musálem S. y G. Vera C. 2002. Radiación fotosintéticamente activa y propiedades físico-químicas en suelos forestales con y sin incendio. Madera y Bosques 8(2):39-55. 